\title{
Modifications of a Nanomolar Cyclic Peptide Antagonist for the EphA4 Receptor To Achieve High Plasma Stability
}

\author{
Erika J. Olson, ${ }^{\dagger, \perp}$ Bernhard C. Lechtenberg, ${ }^{\dagger, \perp}$ Chunxia Zhao, ${ }^{\dagger}$ Elena Rubio de la Torre, ${ }^{\dagger}$
}

Ilaria Lamberto, ${ }^{\dagger, \|}$ Stefan J. Riedl, ${ }^{\dagger}$ Philip E. Dawson, ${ }^{*, \dagger}$ and Elena B. Pasquale ${ }^{*}, \dagger, \S$

$\dagger$ Cancer Center, Sanford Burnham Prebys Medical Discovery Institute, La Jolla, California 92037, United States

${ }^{\ddagger}$ Departments of Chemistry and Cell and Molecular Biology, The Scripps Research Institute, La Jolla, California 92037, United States

${ }^{\S}$ Pathology Department, University of California, San Diego, La Jolla, California 92093, United States

\section{Supporting Information}

ABSTRACT: EphA4 is a receptor tyrosine kinase with a critical role in repulsive axon guidance and synaptic function. However, aberrant EphA4 activity can inhibit neural repair after injury and exacerbate neurodegenerative diseases such as amyotrophic lateral sclerosis (ALS) and Alzheimer's. We previously identified the cyclic peptide APY-d2 (APYCVYR $\beta$ ASWSC- $\mathrm{NH}_{2}$, containing a disulfide bond) as a potent and selective EphA4 antagonist. However, APY-d2 lacks sufficient plasma stability to be useful for EphA4 inhibition in vivo through peripheral administration. Using structure-activity relationship studies, we show that protecting the peptide N-terminus from proteolytic degradation dramatically increases the persistence of the active peptide in plasma and that a positively charged peptide N-terminus is essential for high EphA4 binding affinity. Among several improved APY-d2 derivatives, the cyclic peptides APY-d3 ( $\beta$ APYCVYR $\beta$ ASWSC- $\mathrm{NH}_{2}$ ) and APY-d4 ( $\left.\underline{\beta A P Y C V Y R} \beta A \underline{E} \underline{\underline{E} C}-\mathrm{NH}_{2}\right)$ combine high stability in plasma and cerebrospinal fluid with slightly enhanced potency. These properties make them valuable research tools and leads toward development of therapeutics for neurological diseases.

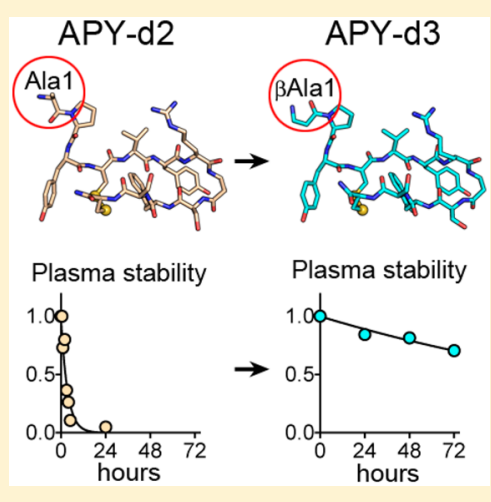

KEYWORDS: Peptide inhibitor, SAR, protease resistance, aminopeptidase, ALS, Alzheimer's disease

EphA4, a member of the large Eph receptor tyrosine kinase family, is predominantly expressed in the nervous system. Through activation by the cell surface-anchored ephrin ligands, EphA4 plays important physiological roles in axon guidance during development as well as in the structural remodeling of synaptic connections and modulation of synaptic transmission in the adult brain. ${ }^{1-3}$ However, increased EphA4 expression and aberrant kinase activity following nervous system injury or disease can contribute to pathological processes. ${ }^{1,3}$ EphA4 was identified as a gene promoting disease pathogenesis in amyotrophic lateral sclerosis (ALS), and studies in ALS animal models and patients have shown that reduced EphA4 activity delays disease onset and slows disease progression. ${ }^{4}$ Evidence in cultured neurons and mouse models has also implicated EphA4 activity in the synaptic dysfunction induced by the pathogenic amyloid- $\beta$ oligomers in Alzheimer's disease. ${ }^{5,6}$ Furthermore, EphA4 is preferentially expressed in breast cancer stem cells, where it supports malignancy by interacting with ephrin ligands expressed by monocytes/macrophages. ${ }^{7}$

Thus, EphA4 is a promising target for promoting neural repair after injury and stroke, counteracting neurodegenerative processes, and inhibiting breast cancer malignancy. ${ }^{1-3}$ The two main strategies that could be used to inhibit the detrimental effects of EphA4 activity involve blocking either its kinase activity or its interaction with ephrin ligands. ${ }^{1,2}$ To our knowledge, high affinity, selective EphA4 kinase inhibitors remain to be identified. ${ }^{1,3}$ In contrast, selective and high affinity peptide antagonists targeting the ephrin-binding pocket in the extracellular ligand-binding domain (LBD) of EphA4 are available. $^{2}$ Peptides appear to have greater potential than small molecules (such as rhynchophylline and bile acid derivatives) for high affinity, selective binding to the ephrinbinding pocket of EphA4 due to its broad surface and conformational flexibility. ${ }^{2,6,8}$ Among the EphA4-targeting peptides identified using phage display screens and other strategies, the linear dodecameric KYL (KYLPYWPVLSSL) was initially considered the most promising. ${ }^{2,9}$ Although the ephrin ligands promiscuously bind multiple Eph receptors, KYL binds only to EphA4. Despite its modest $(\sim 1 \mu \mathrm{M})$ binding affinity, KYL has been instrumental not only as a research tool but also in demonstrating the potential therapeutic value of pharmacologically inhibiting EphA4 in in vitro and in vivo models of ALS, Alzheimer's disease, spinal cord injury, and breast $_{\text {cancer. }}{ }^{4-7,10}$ KYL has been administered in animal models by continuous infusion into the brain or spinal cord with minipumps. ${ }^{4,6,10}$ However, this invasive route of administration has limited applicability in the clinic.

Received: March 28, 2016

Accepted: June 25, 2016

Published: June 25, 2016 
A structurally distinct dodecameric peptide antagonist identified by phage display that also specifically targets EphA4 is APY (APYCVYRGSWSC), which contains a disulfide bond between Cys4 and Cys12. The constrained macrocyclic structure of APY makes it more suitable than KYL for further development toward a therapeutic lead. ${ }^{11-15}$ In previous structure-activity relationship (SAR) studies, APY was modified to obtain a derivative, designated here APY-d2 (APYCVYR $\beta$ ASWSC- $\mathrm{NH}_{2}$, Table 1 ). APY-d2 includes the nonnatural amino acid $\beta \mathrm{Ala}$ at position 8 to stabilize the $\beta$-hairpin geometry within the macrocycle and an amidated $\mathrm{C}$-terminus that provides an additional backbone hydrogen bond in the exocyclic region of the peptide. These modifications increased

Table 1. Potency and Stability of APY Derivatives with NTerminal Modifications

\begin{tabular}{|c|c|c|c|c|}
\hline Name & $\begin{array}{c}\text { Structure } \\
\text { of } \mathrm{N} \text {-terminus }\end{array}$ & Peptide $^{a}$ & $\begin{array}{c}\mathrm{IC}_{50} \pm \mathrm{SEE}(\mathbf{n})^{\mathrm{b}} \\
(\mathrm{nM})^{\mathrm{c}}\end{array}$ & $\begin{array}{c}\mathrm{t}_{1 / 2} \text { of active } \\
\text { peptide in } \\
\text { plasma }^{\mathrm{c}}\end{array}$ \\
\hline $\begin{array}{c}1 \\
\text { APY-d2 }\end{array}$ & & APYCVYR $\beta A S W S C-\mathrm{NH}_{2}$ & $27 \pm 2(25)$ & $<3$ hours \\
\hline 2 & & PYCVYR $\beta A S W S C-\mathrm{NH}_{2}$ & $38 \pm 2(6)$ & $\sim 15$ hours \\
\hline 3 & & YCVYR $\beta A S W S C-\mathrm{NH}_{2} 1$ & $1,200 \pm 300(4)$ & $\mathrm{nd}^{\mathrm{d}}$ \\
\hline 4 & & AC-APYCVYRBASWSC-NH ${ }_{2}$ & $920 \pm 70(3)$ & $\sim 72$ hours \\
\hline 5 & & Ac-PYCVYR $\beta A S W S C-\mathrm{NH}_{2}$ & $450 \pm 100(3)$ & $>72$ hours \\
\hline 6 & & LacPYCVYR $\beta$ ASWSC- $\mathrm{NH}_{2}$ & $230 \pm 50(9)$ & nd \\
\hline 7 & & PEYCVYR $\beta A S W S C-\mathrm{NH}_{2}$ & $290 \pm 20(4)$ & nd \\
\hline 8 & & KYCVYR $\beta A S W S C-\mathrm{NH}_{2}$ & $260 \pm 60(6)$ & nd \\
\hline 9 & & HYCVYR $\beta A S W S C-\mathrm{NH}_{2}$ & $480 \pm 100(3)$ & nd \\
\hline 10 & & AhxYCVYR $\beta A S W S C-\mathrm{NH}_{2}$ & $83 \pm 16(6)$ & $>72$ hours \\
\hline 11 & & AvaYCVYR $\beta A S W S C-\mathrm{NH}_{2}$ & $54 \pm 4(4)$ & $>72$ hours \\
\hline 12 & & $\gamma$ AbuYCVYR $\beta A S W S C-\mathrm{NH}_{2}$ & $34 \pm 7(4)$ & $>72$ hours \\
\hline 13 & & $\beta A Y C V Y R \beta A S W S C-\mathrm{NH}_{2}$ & $25 \pm 4(5)$ & $\sim 30$ hours \\
\hline 14 & & GYCVYR $\beta A S W S C-\mathrm{NH}_{2}$ & $41 \pm 5(4)$ & $\sim 6$ hours \\
\hline 15 & & SarYCVYR $\beta A S W S C-\mathrm{NH}_{2}$ & $40 \pm 9(4)$ & $>72$ hours \\
\hline 16 & & DAPYCVYR $\beta A S W S C-\mathrm{NH}_{2}$ & $50 \pm 4(5)$ & $\sim 72$ hours \\
\hline $\begin{array}{c}17 \\
\text { APY-d3 }\end{array}$ & & $\beta A P Y C V Y R \beta A S W S C-\mathrm{NH}_{2}$ & $19 \pm 2(23)$ & $>72$ hours \\
\hline
\end{tabular}

${ }^{a}$ All peptides are cyclized by a disulfide bond between Cys 4 and Cys12; Ahx, 6-aminohexanoic acid; Ava, 5-aminovaleric acid; $\gamma \mathrm{Abu}, \gamma$ aminobutyric acid; Lac, lactic acid; $\mathrm{PE}$, pyroglutamine; Sar, sarcosine. ${ }^{b}$ Average $\mathrm{IC}_{50}$ value \pm standard error, calculated from $n$ experiments. ${ }^{c}$ Measured by ELISA. ${ }^{d}$ nd $=$ not determined.
EphA4 binding affinity from $1.5 \mu \mathrm{M}$ for APY to $30 \mathrm{nM}$ for APY-d2. ${ }^{15}$

An important step to make peptides suitable for in vivo peripheral administration is to overcome their high susceptibility to proteolytic degradation in the blood circulation. ${ }^{11-14}$ APY-d2 rapidly loses its ability to inhibit ephrin-A5-EphA4 interaction (measured in ELISAs) upon incubation in mouse plasma (half-life of the active peptide $<3 \mathrm{~h}$; Figure $1 \mathrm{~A}$ and Table 1). Thus, we sought to increase the half-life of the active peptide in plasma.

The macrocycle of APY-d2 defined by the disulfide bond forms a $\beta$-hairpin structure that is likely to resist proteolysis, and the C-terminus of the peptide is protected by amidation. ${ }^{15}$ Since exopeptidases are often responsible for the rapid degradation of unstructured peptides in the blood, ${ }^{11-13}$ we focused on modification of the three N-terminal residues, which
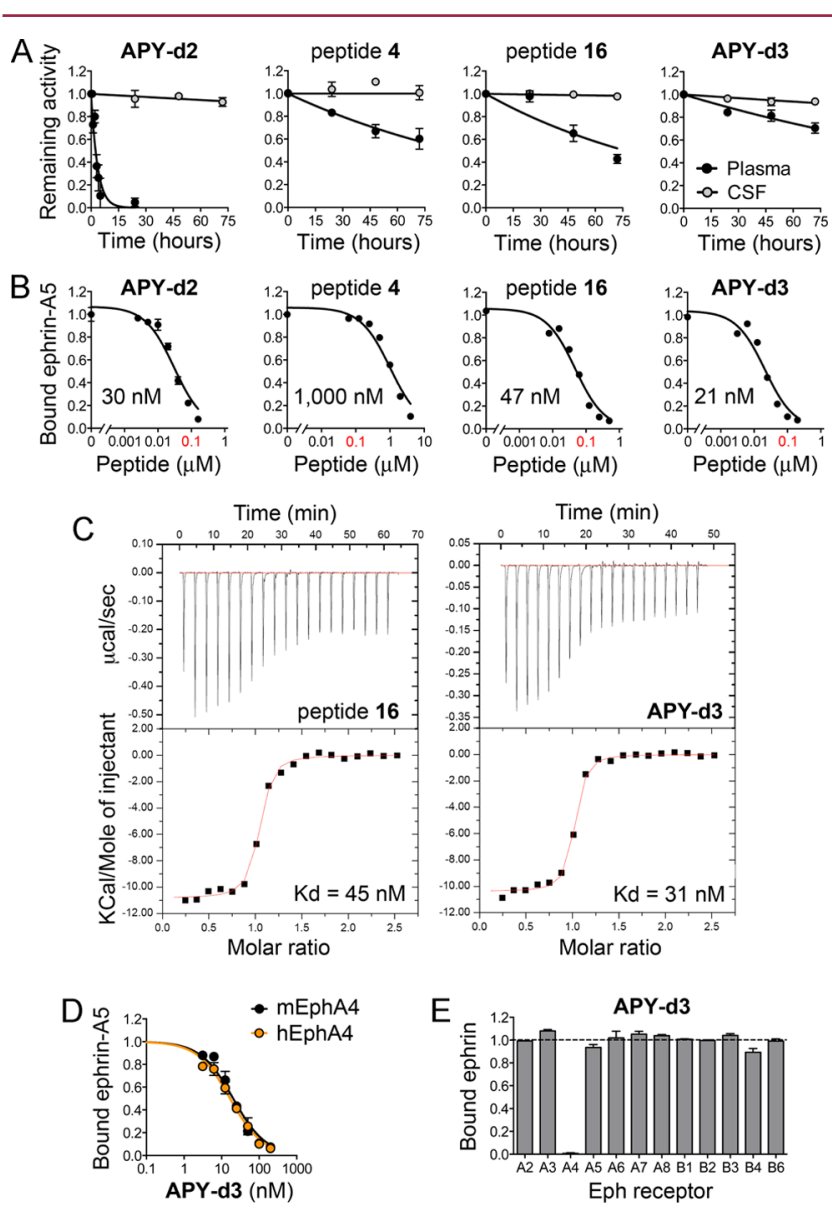

Figure 1. Characterization of APY-d2 derivatives with N-terminal modifications. (A) Peptide antagonistic activity remaining after incubation in mouse plasma or rat CSF for different time periods. (B) Representative ELISA curves measuring peptide inhibition of ephrin-A5-EphA4 interaction; $\mathrm{IC}_{50}$ values for each curve are shown, while averages from multiple experiments are reported in Table 1. (C) Isothermal titration calorimetry profiles (upper panels) and integrated values for the reaction heats (lower panels) versus EphA4/peptide molar ratios. Calculated $K_{\mathrm{d}}$ values are shown. (D) ELISA curves measuring APY-d3 inhibition of ephrin-A5 binding to mouse EphA4 (mEphA4; $\left.\mathrm{IC}_{50}=20 \mathrm{nM}\right)$ and human EphA4 (hEphA4; $\mathrm{IC}_{50}=17$ $\mathrm{nM}$ ). (E) ELISA measuring inhibition of ephrin binding to different Eph receptors. Bound ephrin is the signal in the presence of $2.7 \mu \mathrm{M}$ APY-d3 normalized to the signal without peptide. Averages from triplicate measurements $\pm \mathrm{SE}$ are shown in $\mathrm{A}, \mathrm{B}, \mathrm{D}$, and $\mathrm{E}$. 
are outside the macrocycle. Loss of Ala1 in peptide $\mathbf{2}$ only slightly decreased potency while increasing plasma half-life to $\sim 15 \mathrm{~h}$ (Table 1). However, loss of both Ala1 and Pro2 in peptide 3 decreased potency by nearly 50 -fold (Table 1 ). These results together with the observed dramatic decrease of APY-d2 antagonistic activity in plasma suggest that APY-d2 is inactivated by a dipeptidyl peptidase cleaving the $\mathrm{N}$-terminal Ala-Pro dipeptide. ${ }^{12,13}$

To reduce susceptibility to aminopeptidases, we chemically modified the N-terminus of APY-d2 and peptide 2, its active derivative lacking Ala1. We first explored $\mathrm{N}$-terminal acetylation, which is a widely used strategy to prevent peptide degradation by aminopeptidases. ${ }^{11-13}$ The active acetylated versions of peptides APY-d2 and 2 (derivatives 4 and 5, respectively) both had a greatly increased half-life of $\sim 72 \mathrm{~h}$ in mouse plasma (Figure 1A; Table 1). This indicates that the $\mathrm{N}$ terminus is the main site susceptible to proteolysis and confirms the hypothesis that the disulfide constrained cyclic portion and the C-terminus of the peptides are resistant to plasma peptidases. However, acetylation reduced the antagonistic potency of the peptides by $>30$-fold ( 4 versus APY-d2) and $>10$-fold (5 versus 2) (Figure 1B; Table 1). This drastic reduction in potency was unexpected because the $\mathrm{N}$-terminus of APY-d2 engages in few direct interactions with the EphA4 LBD in the crystallized complex. ${ }^{15}$ The loss in affinity of $\mathbf{5}$ was particularly surprising since this peptide does not contain any chemical moiety that is not present in APY-d2; the acetyl group structurally replaces the carbonyl and $\alpha$-carbon of Alal and thus should not cause destabilizing steric clashes.

These results suggest that the positive charge of the peptide $\mathrm{N}$-terminus is necessary for high affinity interaction with EphA4. To further examine this hypothesis, we substituted the first or the first two amino acids of APY-d2 to place a neutral hydrogen bond donor at the $\mathrm{N}$-terminus (lactyl in 6 and pyroglutamyl in 7; Table 1). We found that both of these neutralized derivatives have $\sim 8$-fold reduced binding affinity $(6$ versus APY-d2 and 7 versus 2), supporting the importance of the $\mathrm{N}$-terminal positive charge. However, introducing an additional $\mathrm{N}$-terminal positive charge by substituting the first two amino acids of APY-d2 with either Lys (8) or His (9) substantially reduced potency, presumably due to steric clashes involving the Lys and His side chains.

To investigate the relationship between potency and the distance of the $\mathrm{N}$-terminal charge from the macrocycle, we examined a series of $\mathrm{N}$-terminal primary amines spaced $2-6$ carbons (corresponding to $\sim 4-8 \AA$ ) from Tyr3 (10-14; Table 1). Furthermore, to discern how the size of the peptide $\mathrm{N}$ terminal charge affects EphA4 binding, we also examined an $\mathrm{N}$ terminal secondary amine (sarcosine in 15; Table 1). All of these derivatives have inhibitory potencies $\left(\mathrm{IC}_{50}\right.$ values) within 0.5-2.5-fold that of 2 (Table 1), indicating that the precise location and size of the positively charged group is not as important as its presence.

While these data identify $\mathbf{1 2}$ and $\mathbf{1 3}$ as having the most favorable combination of potency and plasma stability, neither of these derivatives combines the highest potency with the longest plasma stability. Insights from structure-function analysis suggest that APY-d2 plasma stability can be extended by making the peptide $\mathrm{N}$-terminus resistant to aminopeptidases and that an $\mathrm{N}$-terminal amine is required to maintain high binding affinity. In order to meet both criteria, Ala1 of APY-d2 was substituted with DAla in $\mathbf{1 6}$ and $\beta$ Ala in $\mathbf{1 7}$ (renamed APYd3; Table 1). The active forms of both peptides have a half-life of $>72 \mathrm{~h}$ in mouse plasma (Figure $1 \mathrm{~A}$; Table 1 ). Both peptides also remain active following prolonged incubation in rat cerebrospinal fluid (CSF; Figure 1A), suggesting that they are suitable for cerebroventricular or intrathecal delivery. However, $\beta$ Ala1 in APY-d3 slightly increases potency compared to APYd2, whereas DAla1 in $\mathbf{1 6}$ has the opposite effect (Figure 1B,C; Table 1). Importantly, APY-d3 targets mouse and human EphA4 with similar potency (Figure 1D) and is more than 100fold selective for EphA4 over other Eph receptors (Figure 1E). Thus, although we identified several APY derivatives with favorable properties (including 11, 12, 13, 15, and 16), the high potency, plasma stability, and selectivity of APY-d3, combined with the simple achiral nature of the $\mathrm{N}$-terminal $\beta \mathrm{Ala}$ group, make it the most promising lead for future functional studies.

To obtain structural insights into the interaction of APY-d3 with EphA4, we solved the crystal structure of the APY-d3/ EphA4 LBD complex at $1.75 \AA$ resolution (Figure 2A). The

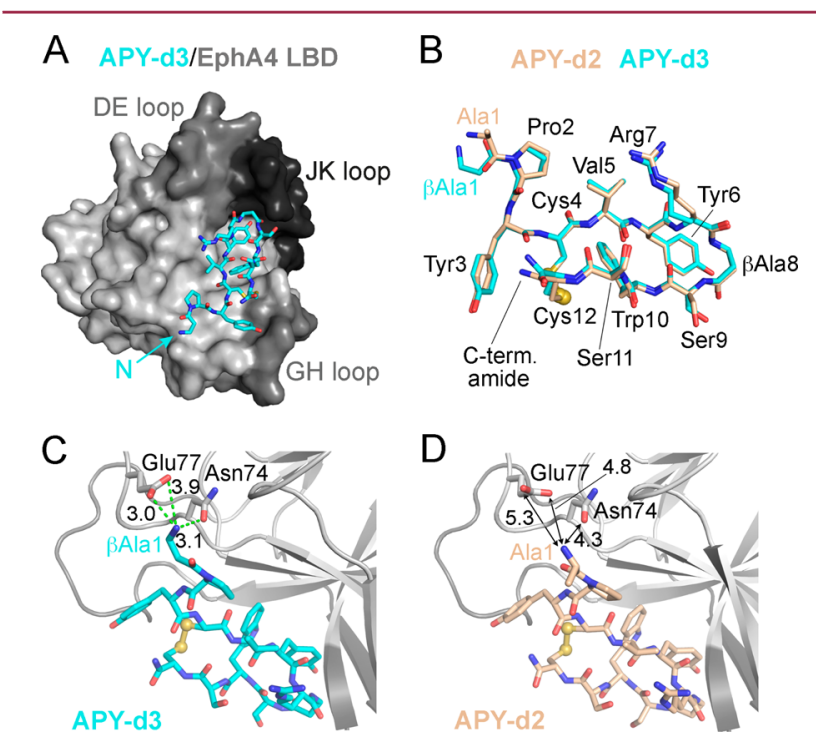

Figure 2. Crystal structure of APY-d3 bound to EphA4. (A) Overall structure of the EphA4 LBD (gray, surface representation with loops surrounding the ephrin-binding pocket in darker gray) in complex with APY-d3 (cyan, sticks; PDB 5JR2). (B) Comparison of APY-d3 (cyan, chain E) and APY-d2 (wheat, chain F of PDB 4W4Z). ${ }^{15}$ (C) Interactions of APY-d3 $\beta$ Alal with EphA4 residues are indicated by green dashes with distances shown in Å. Chains $\mathrm{A}$ and $\mathrm{E}$ are shown. (D) Representation equivalent to $\mathrm{C}$ showing the $\mathrm{N}$-terminus of APYd2. Black arrows (with distances in $\AA$ ) indicate that no direct interactions are present between Ala1 of APY-d2 and EphA4. Chains $\mathrm{B}$ and $\mathrm{F}$ of PDB 4W4Z are shown. All four complexes of the asymmetric unit for both APY-d2 and APY-d3 are shown in Figure S1.

crystallographic asymmetric unit contains four independent EphA4/APY-d3 complexes. APY-d3 adopts the same overall conformation as APY-d2 in the APY-d2/EphA4 complex structure (Figure $2 \mathrm{~B})^{15}$ and thus similarly interacts with the receptor (Figure 2C,D). However, the longer $\beta \mathrm{Alal}$ in APY-d3 enables additional interactions with EphA4 compared to Ala1 in APY-d2. The N-terminus of $\beta$ Ala1 forms a double salt bridge with EphA4 Glu77 and a hydrogen bond with EphA4 Asn74. These interactions are observed in all four EphA4/APY-d3 complexes in the asymmetric unit, although small differences among the four complexes are apparent (Figure S1), indicating conformational flexibility in the peptide/EphA4 interaction. In contrast, the N-terminus of Ala1 in APY-d2 does not make direct interactions with EphA4, as reflected in the long 
distances between the APY-d2 N-terminus and the closest EphA4 residues (Figure 2D; Figure S1). The only exception is a salt bridge between the APY N-terminus and EphA4 Glu77 in one of the four complexes in the asymmetric unit (chains $\mathrm{D} / \mathrm{H}$; Figure S1). Another difference is that in three of the four APYd3 complexes, Pro2 is in the generally thermodynamically less favored cis configuration, whereas all four APY-d2 complexes have Pro2 in the trans configuration (Figure S1). The cis isomer is likely favored due to reduced steric hindrance by $\beta$ Alal, which unlike Ala1 lacks side chain atoms that could clash with cis Pro2. The presence of an alternative backbone suggests that these interactions with EphA4 may be dynamic, which is consistent with the observed SAR.

To examine the importance of the observed salt bridge between the peptide N-terminus and Glu77 for EphA4 binding, we measured the binding affinity of APY-d3 for the EphA4 E77A mutant. Unexpectedly, the E77A mutation had minimal impact on binding affinity (Figure S2A). More detailed analysis of the crystal structure revealed that EphA4 features an extended electronegative surface in the vicinity of the peptide $\mathrm{N}$-terminus, which is still largely preserved in the EphA4 E77A mutant (Figure S2B,C). The redundancy of residues forming the EphA4 electronegative surface could explain the similar potency of peptides that differ substantially in the positioning of the N-terminal amine (Table 1, 10-14). The positional independence of the amino group is probably a function of both the large size of the shallow electronegative surface of EphA4 localized near the $\mathrm{N}$-terminus of the bound peptide and the flexibility of the EphA4 loops that comprise the ephrinbinding pocket.

We also explored further peptide modifications that may increase EphA4 binding affinity or yield a peptide with different charge, which could be more suitable for some applications. Secondary phage display screens previously showed that Ser9 and Ser11 of the original APY peptide are tolerant to substitution. ${ }^{15}$ Furthermore, modeling of APY-d3 with S9E/ S11E substitutions suggested that negative charges at these positions may stabilize the peptide through formation of a salt bridge between the side chains of Glu11 and Arg7 as well as result in an additional salt bridge between the side chains of Glu9 in the peptide and Arg162 in EphA4 (Figure S3). We therefore synthesized an S9E/S11E derivative of APY-d2 (18) as well as an S9D/S11E derivative (19). These peptides, and particularly 18, exhibited slightly increased potency (Table 2 ).

Table 2. Potency and Stability of APY Derivatives with S9 and S11 Modifications

\begin{tabular}{|c|c|c|c|c|}
\hline Name & Peptide $^{a}$ & $\begin{array}{l}\mathrm{IC}_{50} \pm \mathrm{SE}(\mathrm{n})^{\mathrm{b}} \\
\text { by } \operatorname{ELISA}(\mathrm{nM})\end{array}$ & $\begin{array}{l}K_{d} \pm S E(n) \\
\text { by ITC (nM) }\end{array}$ & $\begin{array}{c}\mathbf{t}_{1 / 2} \text { of active } \\
\text { peptide in } \\
\text { plasma }\end{array}$ \\
\hline $\begin{array}{c}1 \\
\text { APY-d2 }\end{array}$ & APYCVYRBASWSC-NH2 & $27 \pm 2(25)$ & $30^{\circ}$ & $<3$ hours \\
\hline 18 & APYCVYR $\beta A E W E C-N H 2^{d}$ & $12 \pm 1(4)$ & $\mathrm{nd}^{\mathrm{e}}$ & nd \\
\hline 19 & APYCVYR $\beta A D W E C-N H 2$ & $22 \pm 8(2)$ & nd & nd \\
\hline $\begin{array}{c}17 \\
\text { APY-d3 }\end{array}$ & $\beta A P Y C V Y R \beta A S W S C-N H 2$ & $19 \pm 2(23)$ & $27 \pm 5(3)$ & $>72$ hours \\
\hline $\begin{array}{c}20 \\
\text { APY-d4 }\end{array}$ & $\beta A P Y C V Y R \beta A E W E C-N H 2$ & $12 \pm 2(5)$ & $19 \pm 1(2)$ & $>72$ hours \\
\hline 21 & $\beta A P Y C V Y R \beta A E E W C-N H 2$ & $>10,000(2)$ & nd & nd \\
\hline
\end{tabular}

${ }^{a}$ All peptides are cyclized by a disulfide bond between Cys4 and Cys12. ${ }^{b}$ Average $\mathrm{IC}_{50}$ value \pm standard error, calculated from $n$ experiments. ${ }^{c}$ From ref $15 .{ }^{d}$ Red font indicates residues that are different from APY-d2 and APY-d3. ${ }^{e}$ nd $=$ not determined.

Thus, we also examined an S9E/S11E derivative of APY-d3 (20, renamed APY-d4), which exhibited slightly better potency and binding affinity than APY-d3 and retained high plasma stability and $>100$-fold selectivity for EphA4 versus other Eph receptors (Figure 3A-D; Table 2). Thus, APY-d4 represents
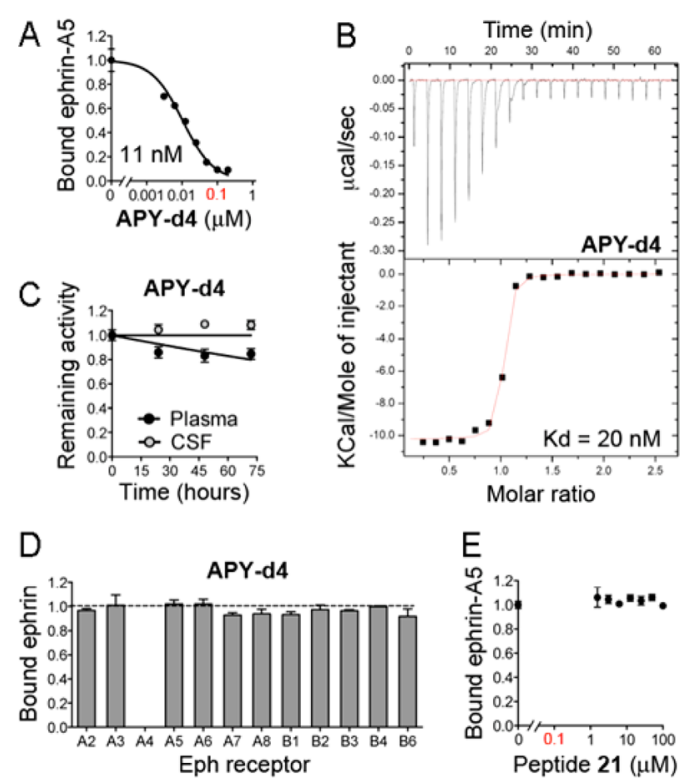

Figure 3. Characterization of APY-d4, a derivative of APY-d3 with S9E and S11E substitutions. (A) Representative ELISA showing inhibition of ephrin-A5 AP binding to EphA4 Fc by APY-d4; the $\mathrm{IC}_{50}$ for the curve is indicated, while the average $\mathrm{IC}_{50}$ is shown in Table 2. (B) Isothermal titration calorimetry profile (upper panel) and integrated values for the reaction heats (lower panel) versus EphA4/ peptide molar ratio. Calculated $K_{d}$ value is shown. (C) APY-d4 antagonistic activity remaining after incubation in mouse plasma or rat CSF. (D) ELISA measuring APY-d4 inhibition of ephrin binding to Eph receptors. Bound ephrin is the signal in the presence of $2 \mu \mathrm{M}$ APY-d4 normalized to the signal without peptide. (E) ELISA measuring inhibition of ephrin-A5-EphA4 interaction by peptide 21 .

an alternative to APY-d3 for EphA4 inhibition. As a strategy to obtain inactive APY-d2 derivatives with very close physicochemical properties to the corresponding active peptides, we explored swapping Trp10 and Glu11, given the importance of Trp at position 10 for EphA4 binding. ${ }^{15}$ We indeed found that a derivative of APY-d4 with Glu10 and Trp11 (21) exhibits at least 1000-fold less activity than APY-d4 (Figure 3E; Table 2).

APY-d3 and APY-d4 represent improved derivatives of APYd2 with greatly increased plasma stability. We confirmed that APY-d3 and APY-d4 inhibit EphA4 tyrosine phosphorylation (activation) induced by ephrin-A5 Fc ligand stimulation in cells (Figure 4A,B) with slightly greater potency than the previously characterized APY-d2. ${ }^{15}$ The higher IC $_{50}$ values for inhibition of ephrin-induced EphA4 phosphorylation in cells compared to inhibition of ephrin binding in the ELISAs are likely due to differences in experimental conditions, and particularly, the higher ephrin concentration needed to activate EphA4 in cells. ${ }^{15}$ Neither peptide showed any evidence of toxicity at concentrations 100-fold higher than their $\mathrm{IC}_{50}$ values for inhibition of EphA4 activation (Figure 4C). Further experiments revealed that APY-d3 effectively inhibits ephrin-induced growth cone collapse in chicken embryo retinal explants at a concentration of $150 \mathrm{nM}$, demonstrating that APY-d3 can 

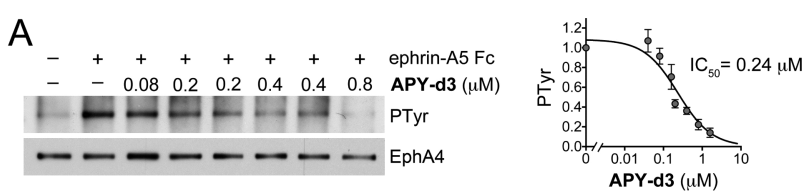

B
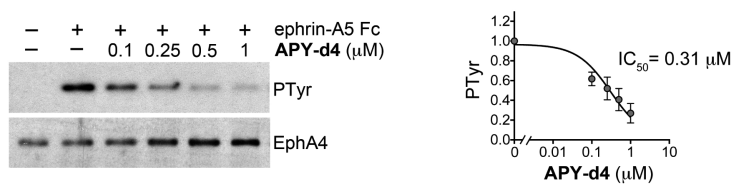

C
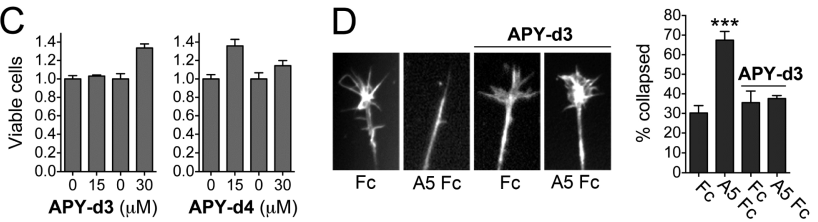

Figure 4. APY-d3 and APY-d4 inhibit EphA4 activation in cells at submicromolar concentrations. $(A, B)$ Inhibition of ephrin-A5-induced EphA4 tyrosine phosphorylation in transfected HEK293 cells by APYd3 (A, with some concentrations run in duplicate) or APY-d4 (B). EphA4 immunoprecipitates were probed for phosphotyrosine (PTyr) and reprobed for EphA4. The graphs show quantification of EphA4 phosphorylation from 10 (A) and 5 (B) experiments, respectively, normalized to the phosphorylation in the ephrin-A5 without peptide condition. Averages from 3 to 6 measurements \pm SE are shown. (C) Cytotoxicity assay. HT22 neuronal cells were grown for $24 \mathrm{~h}$ in the presence of 15 or $30 \mu \mathrm{M}$ peptide, or with the corresponding DMSO concentration ( 0 peptide), and cells were quantified using the MTT assay. Averages from 5 measurements \pm SE are shown. (D) Inhibition of ephrin-induced EphA4-dependent retinal growth cone collapse by $150 \mathrm{nM}$ APY-d3. The histogram shows the average percentages of collapsed growth cones \pm SE $(\sim 13-193$ growth cones per condition in each of 3 experiments). $* * *, P<0.001$ compared to $\mathrm{Fc}$ without peptide by one-way ANOVA and Dunnett's multiple comparisons test.

prevent EphA4-dependent axon repulsion at submicromolar concentrations (Figure 4D), as previously found for APY-d2. ${ }^{15}$

In conclusion, we have investigated the relationship between structure and EphA4-binding capability of APY-d2 derivative peptides. These studies have led to the identification of APY-d3 and APY-d4, two differentially charged derivatives with nanomolar binding affinity and extremely high stability in plasma and CSF. These peptides represent the currently available EphA4 antagonists with the best combination of receptor binding affinity, selectivity, and plasma stability. Future efforts will aim to increase peptide half-life in the blood circulation by decreasing kidney clearance and to achieve peptide delivery to the central nervous system by promoting blood-brain barrier permeability.

\section{ASSOCIATED CONTENT}

\section{S Supporting Information}

The Supporting Information is available free of charge on the ACS Publications website at DOI: 10.1021/acsmedchemlett.6b00132.

Experimental procedures, additional figures, and a table (PDF)

\section{AUTHOR INFORMATION}

\section{Corresponding Authors}

*E-mail: dawson@scripps.edu.

*E-mail: elenap@sbpdiscovery.org.

\section{Present Address}

"Department of Cancer Biology, Dana-Farber Cancer Institute, Boston, Massachusetts 02115, United States.

\section{Author Contributions}

${ }^{\perp}$ These authors (E.J.O. and B.C.L.) contributed equally to this work.

\section{Funding}

This work was supported by NIH grant NS087070 (to E.B.P., S.J.R., P.E.D.), NCI Cancer Center Support Grant P30CA030199 (to E.B.P., S.J.R.), and NSF Graduate Research Fellowship Program DGE-1346837 (E.J.O.).

\section{Notes}

The authors declare no competing financial interest.

\section{ACKNOWLEDGMENTS}

The authors thank A. Bobkov for performing the isothermal titration calorimetry experiments, W. Danho for helpful discussions, and M. Dobaczewska for technical assistance.

\section{ABBREVIATIONS}

ALS, amyotrophic lateral sclerosis; CSF, cerebrospinal fluid; LBD, ligand-binding domain; SAR, structure-activity relationship

\section{REFERENCES}

(1) Barquilla, A.; Pasquale, E. B. Eph receptors and ephrins: Therapeutic opportunities. Annu. Rev. Pharmacol. Toxicol. 2015, 55, 465-487.

(2) Riedl, S. J.; Pasquale, E. B. Targeting the Eph system with peptides and peptide conjugates. Curr. Drug Targets 2015, 16, 10311047.

(3) Boyd, A. W.; Bartlett, P. F.; Lackmann, M. Therapeutic targeting of Eph receptors and their ligands. Nat. Rev. Drug Discovery 2014, 13, $39-62$.

(4) Van Hoecke, A.; Schoonaert, L.; Lemmens, R.; Timmers, M.; Staats, K. A.; Laird, A. S.; Peeters, E.; Philips, T.; Goris, A.; Dubois, B.; Andersen, P. M.; Al-Chalabi, A.; Thijs, V.; Turnley, A. M.; van Vught, P. W.; Veldink, J. H.; Hardiman, O.; Van Den Bosch, L.; GonzalezPerez, P.; Van Damme, P.; Brown, R. H., Jr.; van den Berg, L. H.; Robberecht, W. EphA4 is a disease modifier of amyotrophic lateral sclerosis in animal models and in humans. Nat. Med. 2012, 18, 14181422.

(5) Vargas, L. M.; Leal, N.; Estrada, L. D.; Gonzalez, A.; Serrano, F.; Araya, K.; Gysling, K.; Inestrosa, N. C.; Pasquale, E. B.; Alvarez, A. R. Epha4 activation of $\mathrm{c}$-abl mediates synaptic loss and ltp blockade caused by amyloid-beta oligomers. PLoS One 2014, 9, e92309.

(6) Fu, A. K.; Hung, K. W.; Huang, H.; Gu, S.; Shen, Y.; Cheng, E. Y.; Ip, F. C.; Huang, X.; Fu, W. Y.; Ip, N. Y. Blockade of epha4 signaling ameliorates hippocampal synaptic dysfunctions in mouse models of Alzheimer's disease. Proc. Natl. Acad. Sci. U. S. A. 2014, 111, 9959-9964.

(7) Lu, H.; Clauser, K. R.; Tam, W. L.; Frose, J.; Ye, X.; Eaton, E. N.; Reinhardt, F.; Donnenberg, V. S.; Bhargava, R.; Carr, S. A.; Weinberg, R. A. A breast cancer stem cell niche supported by juxtacrine signalling from monocytes and macrophages. Nat. Cell Biol. 2014, 16, 11051117.

(8) Tognolini, M.; Hassan-Mohamed, I.; Giorgio, C.; Zanotti, I.; Lodola, A. Therapeutic perspectives of Eph-ephrin system modulation. Drug Discovery Today 2014, 19, 661-669.

(9) Murai, K. K.; Nguyen, L. N.; Koolpe, M.; McLennan, R.; Krull, C. E.; Pasquale, E. B. Targeting the EphA4 receptor in the nervous system with biologically active peptides. Mol. Cell. Neurosci. 2003, 24, $1000-1011$

(10) Fabes, J.; Anderson, P.; Brennan, C.; Bolsover, S. Regenerationenhancing effects of EphA4 blocking peptide following corticospinal 
tract injury in adult rat spinal cord. Eur. J. Neurosci 2007, 26, 24962505.

(11) Adessi, C.; Soto, C. Converting a peptide into a drug: Strategies to improve stability and bioavailability. Curr. Med. Chem. 2002, 9, 963-978.

(12) Werle, M.; Bernkop-Schnurch, A. Strategies to improve plasma half life time of peptide and protein drugs. Amino Acids 2006, 30, 351367.

(13) Nestor, J. J., Jr. The medicinal chemistry of peptides. Curr. Med. Chem. 2009, 16, 4399-4418.

(14) Craik, D. J.; Fairlie, D. P.; Liras, S.; Price, D. The future of peptide-based drugs. Chem. Biol. Drug Des. 2013, 81, 136-147.

(15) Lamberto, I.; Lechtenberg, B. C.; Olson, E. J.; Mace, P. D.; Dawson, P. E.; Riedl, S. J.; Pasquale, E. B. Development and structural analysis of a nanomolar cyclic peptide antagonist for the EphA4 receptor. ACS Chem. Biol. 2014, 9, 2787-2795. 